Mathematical Modelling and Analysis

Volume 21 Number 3, May 2016, 350-370

http://dx.doi.org/10.3846/13926292.2016.1167787

(c) Vilnius Gediminas Technical University, 2016
Publisher: Taylor\&Francis and VGTU

http://www.tandfonline.com/TMMA

ISSN: $1392-6292$

eISSN: $1648-3510$

\title{
Application of the Homotopy Analysis Method for Solving the Systems of Linear and Nonlinear Integral Equations
}

\section{Rafał Brociek, Edyta Hetmaniok, Jarosław Matlak and Damian Słota}

\author{
Institute of Mathematics, Silesian University of Technology \\ Kaszubska 23, 44-100 Gliwice, Poland \\ E-mail(corresp.): rafal.brociek@polsl.pl \\ E-mail: edyta.hetmaniok@polsl.pl \\ E-mail: jaroslaw.matlak@polsl.pl \\ E-mail: damian.slota@polsl.pl
}

Received November 2, 2015; revised March 14, 2016; published online May 15, 2016

\begin{abstract}
In this paper we indicate some applications of homotopy analysis method for solving the systems of linear and nonlinear integral equations. The method is based on the concept of creating function series. If the series converges, its sum is the solution of this system of equations. The paper presents conditions to ensure the convergence of this series and estimation of the error of approximate solution obtained when the partial sum of the series is used. Application of the method will be illustrated by examples.
\end{abstract}

Keywords: homotopy analysis method, convergence, error estimation, system of Fredholm integral equations, system of Volterra integral equations.

AMS Subject Classification: 65R20; 45F05.

\section{Introduction}

Homotopy analysis method was developed in the 90s of the last century by Chinese mathematician Shijun Liao $[13,14,15,16,17]$. Using this method we are able to solve the operator equation

$$
N(u(x))=0, \quad x \in \Omega,
$$

where $N$ is an operator (in particular, it may be non-linear operator) and $u$ is an unknown function. In the first step we define the homotopy operator $\mathcal{H}$ as

$$
\mathcal{H}(\Phi, p) \equiv(1-p) L\left(\Phi(x ; p)-u_{0}(x)\right)-p h N(\Phi(x ; p))
$$


where $p \in[0,1]$ is the embedding parameter, $h \neq 0$ is the convergence control parameter, $u_{0}$ denotes the initial approximation of solution of problem (1.1) and $L$ is the auxiliary linear operator with property $L(0)=0$.

Solving equation $\mathcal{H}(\Phi, p)=0$, we get the zero-order deformation equation

$$
(1-p) L\left(\Phi(x ; p)-u_{0}(x)\right)=p h N(\Phi(x ; p)) .
$$

For $p=0$ we get $L\left(\Phi(x ; 0)-u_{0}(x)\right)=0$. It implies that $\Phi(x ; 0)=u_{0}(x)$. For $p=1$ we get $N(\Phi(x ; 1))=0$ and $\Phi(x ; 1)=u(x)$, where $u$ is the solution of equation (1.1). In this way, change of parameter $p$ from zero to one corresponds to the transition from a trivial task to the original task.

By expanding function $\Phi$ in the Maclaurin series with respect to parameter $p$ we get

$$
\Phi(x ; p)=\Phi(x ; 0)+\left.\sum_{m=1}^{\infty} \frac{1}{m !} \frac{\partial^{m} \Phi(x ; p)}{\partial p^{m}}\right|_{p=0} p^{m} .
$$

Denoting

$$
u_{m}(x)=\left.\frac{1}{m !} \frac{\partial^{m} \Phi(x ; p)}{\partial p^{m}}\right|_{p=0}, \quad m=1,2,3, \ldots
$$

we can write the previous relation as follows

$$
\Phi(x ; p)=u_{0}(x)+\sum_{m=1}^{\infty} u_{m}(x) p^{m} .
$$

If the above series possesses the radius of convergence not smaller than one and the series $\sum_{m=0}^{\infty} u_{m}(x)$ is absolutely convergent, then according to Abel's Theorem the solution of equation (1.1) is obtained

$$
u(x)=\sum_{m=0}^{\infty} u_{m}(x) .
$$

In order to determine function $u_{m}$ we differentiate $m$-times the left and the right side of equation (1.2) with respect to parameter $p$. Next, we divide the result by $m$ ! and substitute $p=0$ getting the $m$ th-order deformation equation $(m>0)$ :

$$
L\left(u_{m}(x)-\chi_{m} u_{m-1}(x)\right)=h R_{m}\left(\bar{u}_{m-1}, x\right),
$$

where $\bar{u}_{m-1}=\left\{u_{0}(x), u_{1}(x), \ldots, u_{m-1}(x)\right\}$,

$$
\begin{aligned}
& \chi_{m}= \begin{cases}0 & m \leq 1, \\
1 & m>1,\end{cases} \\
& R_{m}\left(\bar{u}_{m-1}, x\right)=\left.\frac{1}{(m-1) !}\left(\frac{\partial^{m-1}}{\partial p^{m-1}} N\left(\sum_{i=0}^{\infty} u_{i}(x) p^{i}\right)\right)\right|_{p=0} .
\end{aligned}
$$

If we are not able to determine the sum of series in (1.4), then as an approximate solution of considered equation we can accept the partial sum of this series

$$
\widehat{u}_{n}(x)=\sum_{m=0}^{n} u_{m}(x) .
$$


Setting appropriately the value of the convergence control parameter $h$ we can affect the area of convergence of series (1.3) as well as the convergence rate $[17,19,23]$. One method of determining value of the convergence control parameter is the "optimization method" $[3,17,26]$. In this method we define the squared residual of governing equation

$$
E_{n}(h)=\int_{\Omega}\left\|N\left[\widehat{u}_{n}(x)\right]\right\|^{2} d x .
$$

We can obtain the optimal value of the convergence control parameter by calculating the minimum of squared residual (1). Effective region of the convergence control parameter is defined by

$$
\mathbf{R}_{h}=\left\{h: \lim _{n \rightarrow \infty} E_{n}(h)=0\right\} .
$$

By choosing the convergence control parameter value other than optimal (but from the effective region) we get a convergent series as well, only the rate of convergence of the series will be less. More details concerning the effective region and the optimal value of the convergence control parameter are given by the inventor of this method S. Liao in his book [17].

Papers $[2,4,5,6,18,24]$ describe the use of investigated method for solving the integral equations. However, most of these papers describe only examples illustrating the application of homotopy analysis method, mostly without any theoretical analysis. Most often there is given only a theorem showing that if the series converges its sum is the solution of the equation. More theoretical results are contained in papers $[1,10,11,25]$.

Papers $[1,25]$ describe an application of considerd method for nonlinear Fredholm and Volterra integral equation of the second kind. Paper [1] shows that if the series converges its sum is the solution of the Fredholm equation. The same theorem for Volterra equation was proven in paper [25]. Uniqueness of solution of the Volterra equation and the sufficient condition of convergence of the created series are also shown. Paper [11] describes the application of homotopy analysis method for nonlinear and linear integral equations of the second kind. The form of considered equations is more general than the form of equations described in $[1,25]$. In particular, paper [11] shows that the equation has a unique solution (under appropriate assumptions) and proves that if the created series converges its sum is the solution of considered equation. Authors describe also the sufficient condition of convergence of the created series and error estimation of the approximate solution. In paper [11] the authors present also the example which has practical application for charge calculation in supply circuit of flash lamps used in cameras. In paper [10] the nonlinear and linear Volterra-Fredholm integral equation are considered.

Papers $[20,21]$ present the examples of application of the homotopy analysis method for systems of integral equations. However, there is no theoretical results. In the current study we will show that if the created series converges its sum is the solution of considered system of equations. We will also formulate and prove the sufficient condition of convergence of the created series and uniqueness of the solution. Next, we will describe estimation of error of 
the approximate solution obtained when the partial sum of the series is used. Application of the method will be illustrated by examples.

\section{System of linear integral equations}

We consider a system of the integral equations

$$
\mathbf{U}(x)-\int_{f(x)}^{g(x)} \mathbf{K}(x, t) R(\mathbf{U}(t)) d t=\mathbf{F}(x),
$$

where $x \in[a, b]$ and

$$
\begin{gathered}
\mathbf{K}(x, t)=\left[\begin{array}{cccc}
K_{11}(x, t) & K_{12}(x, t) & \ldots & K_{1 n}(x, t) \\
K_{21}(x, t) & K_{22}(x, t) & \ldots & K_{2 n}(x, t) \\
\vdots & \vdots & \ddots & \vdots \\
K_{n 1}(x, t) & K_{n 2}(x, t) & \ldots & K_{n n}(x, t)
\end{array}\right], \\
\mathbf{U}(x)=\left[\begin{array}{c}
u_{1}(x) \\
u_{2}(x) \\
\vdots \\
u_{n}(x)
\end{array}\right], \quad \mathbf{F}(x)=\left[\begin{array}{c}
f_{1}(x) \\
f_{2}(x) \\
\vdots \\
f_{n}(x)
\end{array}\right],
\end{gathered}
$$

where $R:(C[a, b])^{n} \rightarrow(C[a, b])^{n}$ is a bounded linear operator, $f, g \in C[a, b]$, $a \leqslant f(x) \leqslant g(x) \leqslant b$, functions $K_{i j} \in C([a, b] \times[a, b])$ and $f_{i} \in C[a, b]$ are known, whereas the functions $u_{i}$ are sought. Special cases of the above system of equations are the systems of Fredholm and Volterra integral equations of the second kind.

Assume the supremum norm as the norm of function $v \in C(\Omega)$ :

$$
\|v\|=\sup _{x \in \Omega}|v(x)|
$$

For the vector of functions $\mathbf{V}(x)=\left[v_{1}(x), v_{2}(x), \ldots, v_{n}(x)\right]^{T}, v_{i} \in C(\Omega)$ for $i=1,2, \ldots, n$, we define the norm as

$$
\|\mathbf{V}\|=\max \left\{\left\|v_{1}\right\|,\left\|v_{2}\right\|, \ldots,\left\|v_{n}\right\|\right\}
$$

Then it induces the matrix norm of the form

$$
\|\mathbf{A}\|:=\max _{1 \leqslant i \leqslant n} \sum_{j=1}^{n}\left\|a_{i j}\right\| .
$$

The functions $K_{i j}$ and $f_{i}$ are continuous in regions $\Omega_{1}=[a, b] \times[a, b]$ and $\Omega=[a, b]$, respectively. This means that there exist the positive numbers $M_{K}$ and $M_{F}$ such that

$$
\|\mathbf{K}(x, t)\| \leqslant M_{K} \wedge\|\mathbf{F}(x)\| \leqslant M_{F} \quad \text { for all } x, t \in[a, b] .
$$


Operators $L$ and $N$ can be defined in the following way

$$
L(\mathbf{V})=\mathbf{V}, \quad N(\mathbf{V})=\mathbf{V}(x)-\mathbf{F}(x)-\int_{f(x)}^{g(x)} \mathbf{K}(x, t) R(\mathbf{V}(t)) d t .
$$

Let $\mathbf{U}_{0} \in(C[a, b])^{n}$. In this case, after using the homotopy analysis method we get the following formula for function $\mathbf{U}_{m}$ :

$$
\mathbf{U}_{m}(x)=\chi_{m} \mathbf{U}_{m-1}(x)+h R_{m}\left(\overline{\mathbf{U}}_{m-1}, x\right),
$$

where $\mathbf{U}_{m}(x)=\left[u_{m, 1}(x), \ldots, u_{m, n}(x)\right]^{T}, \overline{\mathbf{U}}_{m-1}=\left\{\mathbf{U}_{0}(x), \ldots, \mathbf{U}_{m-1}(x)\right\}, \chi_{m}$ and $R_{m}$ are specified by formulas (1.6).

In considered case the operator $R_{m}$ takes the following form (under assumption that the series is convergent, which will be discussed later):

$$
\begin{gathered}
R_{m}\left(\overline{\mathbf{U}}_{m-1}, x\right)=\frac{1}{(m-1) !}\left(\frac{\partial^{m-1}}{\partial p^{m-1}} N\left(\sum_{i=0}^{\infty} \mathbf{U}_{i}(x) p^{i}\right)\right)_{p=0} \\
=\frac{1}{(m-1) !} \frac{\partial^{m-1}}{\partial p^{m-1}}\left[\sum_{i=1}^{\infty} \mathbf{U}_{i}(x) p^{i}-\mathbf{F}(x)-\int_{f(x)}^{g(x)} \mathbf{K}(x, t) R\left(\sum_{i=1}^{\infty} \mathbf{U}_{i}(t) p^{i}\right) d t\right]_{p=0} \\
=\frac{1}{(m-1) !} \frac{\partial^{m-1}}{\partial p^{m-1}}\left[\sum_{i=1}^{\infty} \mathbf{U}_{i}(x) p^{i}-\mathbf{F}(x)-\sum_{i=1}^{\infty} \int_{f(x)}^{g(x)} \mathbf{K}(x, t) R\left(\mathbf{U}_{i}(t)\right) p^{i} d t\right]_{p=0} \\
=\frac{1}{(m-1) !}\left((m-1) ! \mathbf{U}_{m-1}(x)-\left(1-\chi_{m}\right) \mathbf{F}(x)\right. \\
\left.-\int_{f(x)}^{g(x)} \mathbf{K}(x, t)(m-1) ! R\left(\mathbf{U}_{m-1}(t)\right) d t\right) \\
=\mathbf{U}_{m-1}(x)-\frac{1-\chi_{m}}{(m-1) !} \mathbf{F}(x)-\int_{f(x)}^{g(x)} \mathbf{K}(x, t) R\left(\mathbf{U}_{m-1}(t)\right) d t .
\end{gathered}
$$

Using the formula above and equation (2.4) we obtain the formulas for functions $\mathbf{U}_{m}$ :

$$
\mathbf{U}_{1}(x)=h\left(\mathbf{U}_{0}(x)-\mathbf{F}(x)-\int_{f(x)}^{g(x)} \mathbf{K}(x, t) R\left(\mathbf{U}_{0}(t)\right) d t\right)
$$

and for $m \geqslant 2$ :

$$
\mathbf{U}_{m}(x)=(1+h) \mathbf{U}_{m-1}(x)-h \int_{f(x)}^{g(x)} \mathbf{K}(x, t) R\left(\mathbf{U}_{m-1}(t)\right) d t .
$$

Let us start with presenting the following theorem.

Theorem 1. If the condition given below is fulfilled

$$
\|R\| M_{K}(b-a)<1
$$

then the system of equations (2.1) has at most one solution. 
Proof. Assume that there exist two solutions $\mathbf{U}_{1}$ and $\mathbf{U}_{2}$. Then for every $x \in[a, b]$ we have

$$
\begin{aligned}
\left\|\mathbf{U}_{1}(x)-\mathbf{U}_{2}(x)\right\|=\| \int_{f(x)}^{g(x)} \mathbf{K}(x, t) R\left(\mathbf{U}_{1}(t)\right. & \left.-\mathbf{U}_{2}(t)\right) d t \| \\
& \leqslant\|R\| M_{K}(b-a)\left\|\mathbf{U}_{1}-\mathbf{U}_{2}\right\| .
\end{aligned}
$$

The above inequality is satisfied for all $x \in[a, b]$, thus the following inequality is true

$$
\left\|\mathbf{U}_{1}-\mathbf{U}_{2}\right\| \leqslant\|R\| M_{K}(b-a)\left\|\mathbf{U}_{1}-\mathbf{U}_{2}\right\| .
$$

We get then

$$
\left(1-\|R\| M_{K}(b-a)\right)\left\|\mathbf{U}_{1}-\mathbf{U}_{2}\right\| \leqslant 0 .
$$

Since condition (2.7) is satisfied, the equality $\mathbf{U}_{1}=\mathbf{U}_{2}$ is true.

We will show now that the sum of series generated in the homotopy analysis method is the solution of considered system of integral equations.

Theorem 2. Let the vector-valued functions $\mathbf{U}_{m}, m \geqslant 1$, be defined by formulas (2.5) and (2.6). Thus, if the series in (1.4) is convergent then the sum of this series is the solution of the system of integral equations (2.1).

Proof. Assume that the series in (1.4) is convergent. From the necessary condition for convergence of the series we get that for every $x \in[a, b]$ :

$$
\lim _{m \rightarrow \infty} \mathbf{U}_{m}(x)=0 .
$$

Bearing in mind that we assumed the identity operator as the operator $L$, we have

$$
\begin{aligned}
& \sum_{m=1}^{n} L\left(\mathbf{U}_{m}(x)-\chi_{m} \mathbf{U}_{m-1}(x)\right)=\sum_{m=1}^{n}\left(\mathbf{U}_{m}(x)-\chi_{m} \mathbf{U}_{m-1}(x)\right) \\
& \quad=\mathbf{U}_{1}(x)+\left(\mathbf{U}_{2}(x)-\mathbf{U}_{1}(x)\right)+\ldots+\left(\mathbf{U}_{n}(x)-\mathbf{U}_{n-1}(x)\right)=\mathbf{U}_{n}(x) .
\end{aligned}
$$

Hence

$$
\sum_{m=1}^{\infty} L\left(\mathbf{U}_{m}(x)-\chi_{m} \mathbf{U}_{m-1}(x)\right)=\lim _{n \rightarrow \infty} \mathbf{U}_{n}(x)=0 .
$$

From equation (1.5) we get

$$
h \sum_{m=1}^{\infty} R_{m}\left(\overline{\mathbf{U}}_{m-1}, x\right)=\sum_{m=1}^{\infty} L\left(\mathbf{U}_{m}(x)-\chi_{m} \mathbf{U}_{m-1}(x)\right)
$$

and since $h \neq 0$ we have

$$
\sum_{m=1}^{\infty} R_{m}\left(\overline{\mathbf{U}}_{m-1}, x\right)=0
$$


After some transformation we get

$$
\begin{gathered}
0=\sum_{m=1}^{\infty} R_{m}\left(\overline{\mathbf{U}}_{m-1}, x\right)=\sum_{m=1}^{\infty}\left(\frac { 1 } { ( m - 1 ) ! } \frac { \partial ^ { m - 1 } } { \partial p ^ { m - 1 } } \left[\sum_{i=1}^{\infty} \mathbf{U}_{i}(x) p^{i}-\mathbf{F}(x)\right.\right. \\
\left.\left.-\int_{f(x)}^{g(x)} \mathbf{K}(x, t) R\left(\sum_{i=1}^{\infty} \mathbf{U}_{i}(t) p^{i}\right) d t\right]_{p=0}\right) \\
=\sum_{m=1}^{\infty}\left(\mathbf{U}_{m-1}(x)-\frac{1-\chi_{m}}{(m-1) !} \mathbf{F}(x)-\int_{f(x)}^{g(x)} \mathbf{K}(x, t) R\left(\mathbf{U}_{m-1}(t)\right) d t\right) \\
=\sum_{m=1}^{\infty} \mathbf{U}_{m-1}(x)-\mathbf{F}(x)-\int_{f(x)}^{g(x)} \mathbf{K}(x, t) R\left(\sum_{m=1}^{\infty} \mathbf{U}_{m-1}(t)\right) d t \\
=\mathbf{U}(x)-\mathbf{F}(x)-\int_{f(x)}^{g(x)} \mathbf{K}(x, t) R(\mathbf{U}(t)) d t
\end{gathered}
$$

therefore $\mathbf{U}(x)=\sum_{m=0}^{\infty} \mathbf{U}_{m}(x)$ fulfills the considered system of integral equations.

In the next theorem we formulate the sufficient condition for the convergence of considered series.

Theorem 3. If inequality (2.7) is fulfilled then the value of the convergence control parameter can be selected so that the series in (1.4) converges uniformly in the interval $[a, b]$.

Proof. Let $\mathbf{U}_{0}$ be the vector of functions from the $C[a, b]$ class. Therefore there exists a positive constant $M_{0}$ such that for every $x \in[a, b]$ we have

$$
\left\|\mathbf{U}_{0}(x)\right\| \leqslant M_{0} .
$$

We seek the constraints for the vector-valued functions $\mathbf{U}_{m}$ in the interval $[a, b]$ :

$$
\begin{aligned}
\left\|\mathbf{U}_{1}(x)\right\| & =\left\|h\left(\mathbf{U}_{0}(x)-\mathbf{F}(x)-\int_{f(x)}^{g(x)} \mathbf{K}(x, t) R\left(\mathbf{U}_{0}(t)\right) d t\right)\right\| \\
& \leqslant|h|\left(\left\|\mathbf{U}_{0}(x)\right\|+\|\mathbf{F}(x)\|+\int_{f(x)}^{g(x)}\|\mathbf{K}(x, t)\|\left\|R\left(\mathbf{U}_{0}(t)\right)\right\| d t\right) \\
& \leqslant|h|\left(M_{0}+M_{F}+M_{K}\|R\| M_{0}(b-a)\right)=: \alpha, \\
\left\|\mathbf{U}_{2}(x)\right\| & =\left\|(1+h) \mathbf{U}_{1}(x)-h \int_{f(x)}^{g(x)} \mathbf{K}(x, t) R\left(\mathbf{U}_{1}(t)\right) d t\right\| \\
& \leqslant\left(|1+h|+|h| M_{K}\|R\|(b-a)\right) \alpha=\beta_{h} \alpha,
\end{aligned}
$$

where

$$
\beta_{h}:=|1+h|+|h| M_{K}\|R\|(b-a) .
$$

Using the above formula it is easy to show by induction that for $m \geqslant 1$ and for every $x \in[a, b]$ we obtain

$$
\left\|\mathbf{U}_{m}(x)\right\| \leqslant \alpha \beta_{h}^{m-1} .
$$


In this way we have the estimation for the considered series

$$
\sum_{m=0}^{\infty} \mathbf{U}_{m}(x) \leqslant \sum_{m=0}^{\infty}\left\|\mathbf{U}_{m}(x)\right\| \leqslant M_{0}+\alpha \sum_{m=1}^{\infty} \beta_{h}^{m-1} .
$$

The last series in the above estimation is a geometric series with common ratio $\beta_{h}$. If $\beta_{h}<1$ (we have $\beta_{h}>0$ ), then under comparison criterion the considered series converges uniformly in the interval $[a, b]$.

Let us see whether it is possible to take parameter $h$ such that $\beta_{h}<1$, i.e.

$$
|1+h|+|h| M_{K}\|R\|(b-a)<1 .
$$

Since $h \neq 0$ the last inequality is equivalent to condition

$$
M_{K}\|R\|(b-a)<\frac{1-|1+h|}{|h|} .
$$

The function on the right side of this inequality takes the following values

$$
\frac{1-|1+h|}{|h|}= \begin{cases}-1-\frac{2}{h} & \text { for } h<-1, \\ 1 & \text { for } h \in[-1,0), \\ -1 & \text { for } h>0 .\end{cases}
$$

So, if condition (2.7) is satisfied then we take the value of parameter $h$ such that the inequality (2.8) is satisfied (in order to do this it is sufficient to take any $h \in[-1,0))$ and then $\beta_{h}<1$.

Remark 1. Theorems from above imply that if condition (2.7) is satisfied then the considered system of integral equations has exactly one solution.

Remark 2. In special case for $h=-1$ and the Volterra equation of the second kind (it means if $f(x)=a$ and $g(x)=x$ for $x \in[a, b]$ ) the series in (1.4) is always convergent (even if inequality (2.7) is not fulfilled). It results from the fact that in this case we obtain the following estimation (see also [12]):

$$
\left\|\mathbf{U}_{m}(x)\right\| \leqslant \alpha \frac{\left(M_{K}\|R\|(x-a)\right)^{m-1}}{(m-1) !}, \quad x \in[a, b], \quad m \geqslant 1 .
$$

Let us move to the proof of theorem giving the estimation of error of the approximate solution.

Theorem 4. If inequality (2.7) is fulfilled and $n \in \mathbb{N}$, then we get the following estimation of error of the approximate solution

$$
\left\|\mathbf{U}(x)-\widehat{\mathbf{U}}_{n}(x)\right\| \leqslant \frac{\beta_{h}^{n}}{1-\beta_{h}} \alpha
$$

for every $x \in[a, b]$, where $\alpha=|h|\left(M_{0}+M_{F}+M_{0} M_{K}\|R\|(b-a)\right)$ and $\beta_{h}=$ $|1+h|+|h| M_{K}\|R\|(b-a)$. 
Proof. Let $n \in \mathbb{N}$. Then for every $x \in[a, b]$ we get

$$
\begin{aligned}
\left\|\mathbf{U}(x)-\widehat{\mathbf{U}}_{n}(x)\right\|=\left\|\sum_{m=n+1}^{\infty} \mathbf{U}_{m}(x)\right\| \\
\qquad \sum_{m=n+1}^{\infty}\left\|\mathbf{U}_{m}(x)\right\| \leqslant \alpha \sum_{m=n+1}^{\infty} \beta_{h}^{m-1}=\frac{\beta_{h}^{n}}{1-\beta_{h}} \alpha .
\end{aligned}
$$

Remark 3. If in the considered method we take $h=-1$ and $\mathbf{U}_{0}(x)=0$ or $\mathbf{U}_{0}(x)=\mathbf{F}(x)$ then it will be equivalent to the method of successive approximations and the Adomian decomposition method (when $\mathbf{U}_{0}(x)=0$ after skipping the first element which is identically equal to zero; see also [1,25]). For $h=-1$ the considered method agrees with the homotopy perturbation method $($ see $[9,12])$.

Remark 4. We can also find some other expressions for the homotopy operator $[14,17]$, i.e.

$$
\mathcal{H}(\Phi, p) \equiv(1-p) L\left(\Phi(x ; p)-u_{0}(x)\right)-p h H(x) N(\Phi(x ; p)),
$$

where $H$ is the auxiliary function. In this case all results obtained in this paper remain true as well. Only the formulas for constants $\alpha$ and $\beta_{h}$ change.

\section{System of nonlinear integral equations}

Now we will consider a system of nonlinear integral equations

$$
\mathbf{U}(x)-\int_{f(x)}^{g(x)} \mathbf{K}(x, t) R(\mathbf{U}(t)) d t=\mathbf{F}(x),
$$

where $x \in[a, b]$, functions $\mathbf{K}, \mathbf{F}$ and $\mathbf{U}$ are defined in the same way as in the previous section, $R:(C[a, b])^{n} \rightarrow(C[a, b])^{n}$ is the nonlinear operator, $f, g \in C[a, b], a \leqslant f(x) \leqslant g(x) \leqslant b$. Similarly as before we define the constants $M_{K}$ and $M_{F}$ (see formula $(2.2)$ ). We assume that $R$ is an operator satisfying the Lipschitz condition

$$
\left\|R\left(\mathbf{V}_{1}\right)-R\left(\mathbf{V}_{2}\right)\right\| \leqslant s\left\|\mathbf{V}_{1}-\mathbf{V}_{2}\right\| \quad \text { for every } \quad \mathbf{V}_{1}, \mathbf{V}_{2} \in(C[a, b])^{n}
$$

and some $s>0$.

Operators $L$ and $N$ are defined by relation (2.3). Taking $\mathbf{U}_{0} \in(C[a, b])^{n}$, after using the homotopy analysis method we get the following formula for function $\mathbf{U}_{m}$ :

$$
\mathbf{U}_{m}(x)=\chi_{m} \mathbf{U}_{m-1}(x)+h R_{m}\left(\overline{\mathbf{U}}_{m-1}, x\right),
$$

where $\mathbf{U}_{m}(x)=\left[u_{m, 1}(x), \ldots, u_{m, n}(x)\right]^{T}, \overline{\mathbf{U}}_{m-1}=\left\{\mathbf{U}_{0}(x), \ldots, \mathbf{U}_{m-1}(x)\right\}, \chi_{m}$ and $R_{m}$ are specified by formulas (1.6). 
Now we obtain the formulas for functions $\mathbf{U}_{m}$ in the following form

$$
\mathbf{U}_{1}(x)=h\left(\mathbf{U}_{0}(x)-\mathbf{F}(x)-\int_{f(x)}^{g(x)} \mathbf{K}(x, t) R\left(\mathbf{U}_{0}(t)\right) d t\right)
$$

and for $m \geqslant 2$

$$
\begin{aligned}
\mathbf{U}_{m}(x) & =(1+h) \mathbf{U}_{m-1}(x) \\
& -\frac{h}{(m-1) !} \int_{f(x)}^{g(x)} \mathbf{K}(x, t)\left(\frac{\partial^{m-1}}{\partial p^{m-1}} R\left(\sum_{i=0}^{\infty} \mathbf{U}_{i}(t) p^{i}\right)\right)_{p=0} d t .
\end{aligned}
$$

One can find in some literature the expression $\left(\frac{\partial^{m-1}}{\partial p^{m-1}} R\left(\sum_{i=0}^{\infty} \mathbf{U}_{i}(t) p^{i}\right)\right)_{p=0}$ computed for various nonlinear operators $R$ (see for example [17]).

Similarly as in the linear case we can prove the theorem stating the uniqueness of solution of considered equation.

Theorem 5. If the following condition is fulfilled

$$
s M_{K}(b-a)<1
$$

then the system of equations (3.1) has at most one solution.

Proof. Proof of this theorem runs analogically as the proof of Theorem 1.

The following theorem is equivalent to Theorem 2 for the case of nonlinear equations.

Theorem 6. Let the vector-valued functions $\mathbf{U}_{m}, m \geqslant 1$, be defined by formulas (3.2) and (3.3). Therefore if $s<1$ and the series in (1.4) is convergent then the sum of this series is the solution of the system of integral equations (3.1).

Proof. Let series in (1.4) be convergent. From the necessary condition for convergence of the series we get that for any $x \in[a, b]$ :

$$
\lim _{m \rightarrow \infty} \mathbf{U}_{m}(x)=0 .
$$

Let us designate

$$
\mathbf{H}_{m}(x)=\left.\frac{1}{m !}\left(\frac{\partial^{m}}{\partial p^{m}} R\left(\sum_{i=0}^{+\infty} \mathbf{U}_{i}(x) p^{i}\right)\right)\right|_{p=0}
$$

If $R$ is the contraction mapping $(s<1)$ and series in $(1.4)$ is convergent to $\mathbf{U}(x)$, then series $\sum_{m=0}^{\infty} \mathbf{H}_{m}(x)$ is convergent to $R(\mathbf{U}(x))$ (it results from [7]).

Using definition of operator $L$ we have

$$
\sum_{m=1}^{n} L\left(\mathbf{U}_{m}(x)-\chi_{m} \mathbf{U}_{m-1}(x)\right)=\mathbf{U}_{n}(x) .
$$


Hence

$$
\sum_{m=1}^{\infty} L\left(\mathbf{U}_{m}(x)-\chi_{m} \mathbf{U}_{m-1}(x)\right)=\lim _{n \rightarrow \infty} \mathbf{U}_{n}(x)=0 .
$$

From equation (1.5) we get

$$
h \sum_{m=1}^{\infty} R_{m}\left(\overline{\mathbf{U}}_{m-1}, x\right)=\sum_{m=1}^{\infty} L\left(\mathbf{U}_{m}(x)-\chi_{m} \mathbf{U}_{m-1}(x)\right)
$$

and since $h \neq 0$ we have

$$
\sum_{m=1}^{\infty} R_{m}\left(\overline{\mathbf{U}}_{m-1}, x\right)=0
$$

After some transformation we get

$$
\begin{aligned}
0= & \sum_{m=1}^{\infty} R_{m}\left(\overline{\mathbf{U}}_{m-1}, x\right)=\sum_{m=1}^{\infty}\left(\frac { 1 } { ( m - 1 ) ! } \frac { \partial ^ { m - 1 } } { \partial p ^ { m - 1 } } \left[\sum_{i=1}^{\infty} \mathbf{U}_{i}(x) p^{i}-\mathbf{F}(x)\right.\right. \\
& \left.\left.\quad-\int_{f(x)}^{g(x)} \mathbf{K}(x, t) R\left(\sum_{i=1}^{\infty} \mathbf{U}_{i}(t) p^{i}\right) d t\right]_{p=0}\right) \\
= & \sum_{m=1}^{\infty}\left(\mathbf{U}_{m-1}(x)-\frac{1-\chi_{m}}{(m-1) !} \mathbf{F}(x)\right. \\
& \left.\quad-\int_{f(x)}^{g(x)} \mathbf{K}(x, t)\left[\frac{1}{(m-1) !} \frac{\partial^{m-1}}{\partial p^{m-1}} R\left(\sum_{i=1}^{\infty} \mathbf{U}_{i}(t) p^{i}\right)\right]_{p=0} d t\right) \\
= & \sum_{m=1}^{\infty}\left(\mathbf{U}_{m-1}(x)-\frac{1-\chi_{m}}{(m-1) !} \mathbf{F}(x)-\int_{f(x)}^{g(x)} \mathbf{K}(x, t) \mathbf{H}_{m-1}(t) d t\right) \\
= & \sum_{m=1}^{\infty} \mathbf{U}_{m-1}(x)-\mathbf{F}(x)-\int_{f(x)}^{g(x)} \mathbf{K}(x, t) \sum_{m=1}^{\infty} \mathbf{H}_{m-1}(t) d t \\
= & \mathbf{U}(x)-\mathbf{F}(x)-\int_{f(x)}^{g(x)} \mathbf{K}(x, t) R(\mathbf{U}(t)) d t .
\end{aligned}
$$

We intend now to formulate the sufficient condition for convergence of the discussed series.

Theorem 7. If we select parameter $h$ in such a way that the constants $\gamma_{h} \in$ $(0,1)$ and $k_{0} \in \mathbb{N}$ exist such that for each $k \geqslant k_{0}$ the following inequality

$$
\left\|\mathbf{U}_{k+1}\right\| \leqslant \gamma_{h}\left\|\mathbf{U}_{k}\right\|
$$

holds true, then the series in (1.4) is uniformly convergent in the interval $[a, b]$. 
Proof. Let $S_{n}$ denote the partial sum of investigated series

$$
S_{n}=\sum_{m=0}^{n} \mathbf{U}_{m}(x) .
$$

Our aim is to reveal that sequence $\left\{S_{n}\right\}$ is the Cauchy sequence. Let us begin by estimating the following norm

$$
\left\|S_{n}-S_{n-1}\right\|=\left\|\mathbf{U}_{n}\right\| \leqslant \gamma_{h}\left\|\mathbf{U}_{n-1}\right\| \leqslant \ldots \leqslant \gamma_{h}^{n-k_{0}}\left\|\mathbf{U}_{k_{0}}\right\| .
$$

Now for any $n, k \in \mathbb{N}, n \geqslant k \geqslant k_{0}$, we get

$$
\begin{aligned}
& \left\|S_{n}-S_{k}\right\| \leqslant\left\|S_{n}-S_{n-1}\right\|+\ldots+\left\|S_{k+1}-S_{k}\right\| \\
& \quad \leqslant \gamma_{h}^{n-k_{0}}\left\|\mathbf{U}_{k_{0}}\right\|+\ldots+\gamma_{h}^{k+1-k_{0}}\left\|\mathbf{U}_{k_{0}}\right\| \\
& \quad=\gamma_{h}^{k+1-k_{0}}\left(\gamma_{h}^{n-k-1}+\ldots+\gamma_{h}+1\right)\left\|\mathbf{U}_{k_{0}}\right\|=\gamma_{h}^{k+1-k_{0}} \frac{1-\gamma_{h}^{n-k}}{1-\gamma_{h}}\left\|\mathbf{U}_{k_{0}}\right\| .
\end{aligned}
$$

Since $\gamma_{h} \in(0,1)$, we conclude that sequence $\left\{S_{n}\right\}$ is the Cauchy sequence. From the completeness of space $\mathbb{R}$ we deduce the convergence of this sequence, which implies in turn the convergence of the investigated series.

In the next theorem we present the estimation of error of approximate solution $\widehat{\mathbf{U}}_{n}$.

Theorem 8. If the assumptions of Theorem 7 are fulfilled and, in addition, $n \in \mathbb{N}$ and $n \geqslant k_{0}$ then we derive the following estimation of error of the approximate solution

$$
\left\|\mathbf{U}-\widehat{\mathbf{U}}_{n}\right\| \leqslant \frac{\gamma_{h}^{n+1-k_{0}}}{1-\gamma_{h}}\left\|\mathbf{U}_{k_{0}}\right\| .
$$

Proof. Let $n \in \mathbb{N}$ and $n \geqslant k_{0}$. Then we receive for every $x \in[a, b]$ :

$$
\begin{aligned}
\left\|\mathbf{U}(x)-\widehat{\mathbf{U}}_{n}(x)\right\| & =\left\|\sum_{m=n+1}^{\infty} \mathbf{U}_{m}(x)\right\| \\
& \leqslant \sum_{m=n+1}^{\infty}\left\|\mathbf{U}_{m}(x)\right\| \leqslant \sum_{m=n+1}^{\infty} \gamma_{h}^{m-k_{0}}\left\|\mathbf{U}_{k_{0}}\right\|=\frac{\gamma_{h}^{n+1-k_{0}}}{1-\gamma_{h}}\left\|\mathbf{U}_{k_{0}}\right\| .
\end{aligned}
$$

\section{Examples}

Example 1. In the first example we apply the investigated method for solving the following system of Fredholm integral equations of the second kind

$$
\begin{aligned}
& u_{1}(x)=-\frac{x^{3}}{12}+\frac{9 x^{2}}{8}-\frac{x}{20}++\frac{1}{4}\left(\int_{0}^{1}(x-t)^{3} u_{1}(t) d t+\int_{0}^{1}(x-t)^{2} u_{2}(t) d t\right) \\
& u_{2}(x)=\frac{15 x^{3}}{16}+\frac{x^{2}}{15}-\frac{1}{70}+\frac{1}{4}\left(\int_{0}^{1}(x-t)^{2} u_{1}(t) d t+\int_{0}^{1}(x-t)^{3} u_{2}(t) d t\right)
\end{aligned}
$$


for $x \in[0,1]$. Solution of the considered system is given by functions

$$
u_{d 1}(x)=x^{2}, \quad u_{d 2}(x)=x^{3} .
$$

In this example we have $M_{K}=\frac{1}{2}$ and $\|R\|=1$. It means that for the discussed system of integral equations the condition (2.7) is satisfied which implies the convergence of homotopy analysis method.

As the initial approximation we take $\mathbf{U}_{0}(x)=[0,0]^{T}$. Thus we obtain

$$
\begin{aligned}
\mathbf{V}_{1}(x)= & {\left[h\left(\frac{x}{20}-\frac{9 x^{2}}{8}+\frac{x^{3}}{12}\right), h\left(\frac{1}{70}-\frac{x^{2}}{15}-\frac{15 x^{3}}{16}\right)\right]^{T}, } \\
\mathbf{V}_{2}(x)= & {\left[h\left(\frac{x}{20}-\frac{9 x^{2}}{8}+\frac{x^{3}}{12}\right)+h^{2}\left(-\frac{13}{67200}+\frac{45 x}{448}-\frac{100829 x^{2}}{80640}+\frac{53 x^{3}}{320}\right),\right.} \\
& \left.h\left(\frac{1}{70}-\frac{x^{2}}{15}-\frac{15 x^{3}}{16}\right)+h^{2}\left(\frac{1}{35}-\frac{23 x}{67200}-\frac{37 x^{2}}{280}-\frac{14143 x^{3}}{16128}\right)\right]^{T}, \ldots
\end{aligned}
$$

In Figure 1 the plot of logarithm of squared residual $E_{3}$ is shown. In this case optimum value of the convergence control parameter is equal to $-1(h=-1)$.

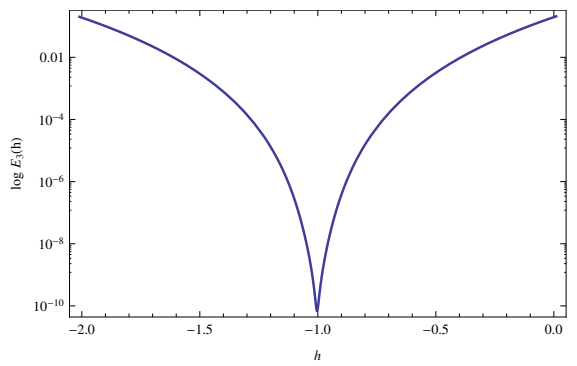

Figure 1. Logarithm of squared residual $E_{3}(h)$

Displayed results show that in the discussed case the approximated effective region of the convergence control parameter, determined on the basis of square residual, is the interval $\mathbf{R}_{h}=(-1.9,0)$ (see Figure $2 \mathrm{~b}$ ). Whereas the approximation of this region determined on the basis of $h$-curve is the smaller interval (see Figure 2a).

As the approximate solution $\widehat{\mathbf{U}}_{n}=\left[\widehat{u}_{1, n}, \widehat{u}_{2, n}\right]^{T}$ defined by sum (1.7) for $h=-1$ and $n=2$ we receive

$$
\begin{aligned}
& \widehat{u}_{1,2}(x)=-0.000193452+0.000446429 x+0.99964 x^{2}-0.00104167 x^{3}, \\
& \widehat{u}_{2,2}(x)=-0.000342262 x+0.00119048 x^{2}+0.998078 x^{3},
\end{aligned}
$$

for $n=5$ we obtain

$$
\begin{aligned}
& \widehat{u}_{1,5}(x)=-8.67362 \cdot 10^{-19}-4.88943 \cdot 10^{-8} x+x^{2}-9.77859 \cdot 10^{-8} x^{3}, \\
& \widehat{u}_{2,5}(x)=-1.42822 \cdot 10^{-8}+4.38272 \cdot 10^{-10} x+5.8059 \cdot 10^{-8} x^{2}+x^{3},
\end{aligned}
$$

whereas for $n=10$ we get

$$
\begin{aligned}
& \widehat{u}_{1,10}(x)=-6.10623 \cdot 10^{-16}+1.42109 \cdot 10^{-14} x+x^{2}+1.42109 \cdot 10^{-14} x^{3}, \\
& \widehat{u}_{2,10}(x)=-1.55431 \cdot 10^{-15} x-2.84217 \cdot 10^{-14} x^{2}+x^{3} .
\end{aligned}
$$




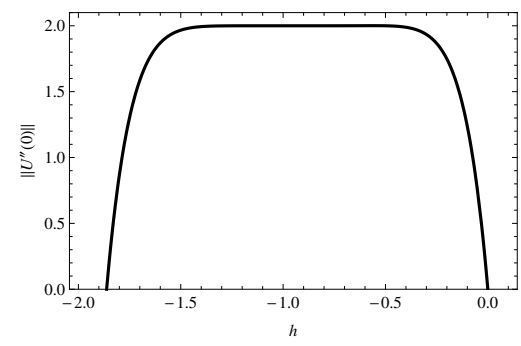

a)

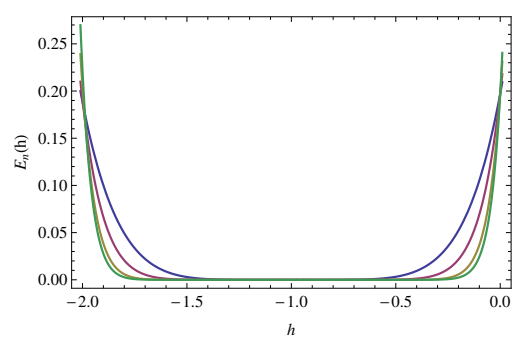

b)

Figure 2. The $h$-curve $(a)$ and squared residual $E_{n}(h)(b)$ for $n=3,5,8,10$ (the successive curves from the top)

All calculations were executed with the aid of computational software Mathematica.

In Table 1 there are presented the errors $\left(\left\|u_{d i}-\widehat{u}_{i, n}\right\|=\sup _{x \in[0,1]} \mid u_{d i}(x)-\right.$ $\left.\widehat{u}_{i, n}(x) \mid\right)$ which occur in approximating the exact solution by the successive approximate solutions. Whereas, distributions of error in the entire interval $[0,1]$ for $n=3$ and $n=9$ are displayed in Figure 3 and Figure 4. Presented results indicate that the method is fast convergent and computing just a few (a dozen or so) first terms of the series ensures a very good approximation of the exact solution.

Table 1. Errors of approximations of the exact solution

\begin{tabular}{llllll}
\hline$n$ & $\left\|u_{d 1}-\widehat{u}_{1, n}\right\|$ & $\left\|u_{d 2}-\widehat{u}_{2, n}\right\|$ & $n$ & $\left\|u_{d 1}-\widehat{u}_{1, n}\right\|$ & $\left\|u_{d 2}-\widehat{u}_{2, n}\right\|$ \\
\hline 1 & $8.333310^{-3}$ & $1.428610^{-2}$ & 6 & $1.249710^{-9}$ & $1.155510^{-9}$ \\
2 & $1.148310^{-3}$ & $1.073910^{-3}$ & 7 & $2.895110^{-11}$ & $3.173310^{-11}$ \\
3 & $1.508810^{-5}$ & $1.690810^{-5}$ & 8 & $1.373010^{-12}$ & $1.266610^{-12}$ \\
4 & $1.176010^{-6}$ & $1.091510^{-6}$ & 9 & $3.592910^{-14}$ & $3.930510^{-14}$ \\
5 & $2.209210^{-8}$ & $2.436510^{-8}$ & 10 & $1.534910^{-15}$ & $3.229010^{-15}$ \\
\hline
\end{tabular}

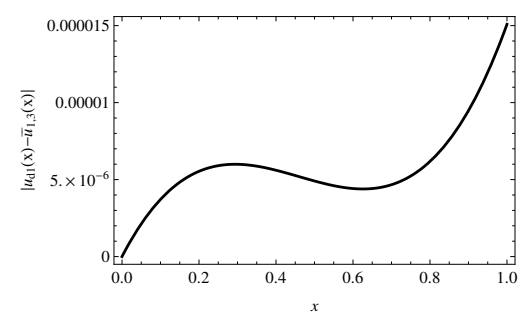

a)

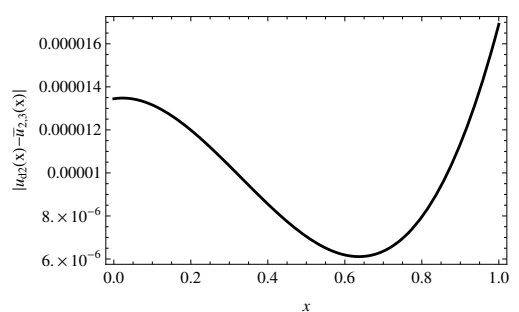

b)

Figure 3. Distribution of error of the exact solution approximation for $n=3$

In general, the convergence is much more faster than the one expected in Theorem 4, in the sense that the error of determined approximate solution is 


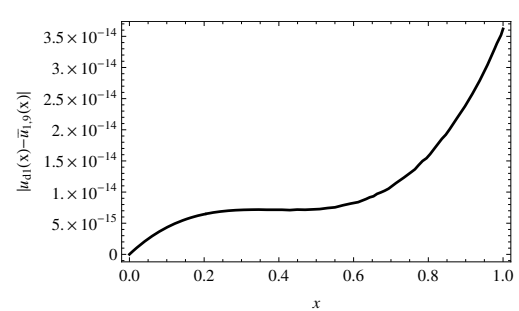

a)

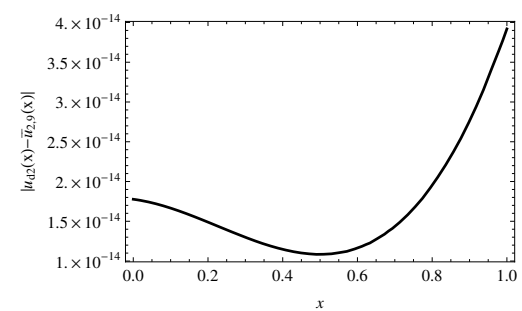

b)

Figure 4. Distribution of error of the exact solution approximation for $n=9$

much lower than its estimation resulting from inequality (2.9). In considered example we have $M_{k}=\frac{1}{2}, M_{0}=0, M_{F}=\frac{119}{120},\|R\|=1, a=0, b=1$. Thereby for the optimal value of convergence control parameter $h=-1$ we obtain

$$
\begin{aligned}
\beta_{h} & =|1+h|+|h| M_{K}\|R\|(b-a)=\frac{1}{2}, \\
\alpha & =|h|\left(M_{0}+M_{F}+M_{0} M_{K}\|R\|(b-a)\right)=\frac{119}{120},
\end{aligned}
$$

and

$$
\left\|\mathbf{U}(x)-\widehat{\mathbf{U}}_{n}(x)\right\| \leqslant \frac{\beta_{h}^{n}}{1-\beta_{h}} \alpha=\frac{119}{120}\left(\frac{1}{2}\right)^{n-1}=: \Delta_{n} .
$$

The estimations of error of the successive approximate solutions, resulting from the above relation, are presented in Table 2 .

Table 2. Estimation $\left(\Delta_{n}\right)$ of the error resulting from relation (2.9)

\begin{tabular}{llll}
\hline$n$ & $\Delta_{n}$ & $n$ & $\Delta_{n}$ \\
\hline 1 & 0.9917 & 6 & $3.099010^{-2}$ \\
2 & 0.4958 & 7 & $1.549510^{-2}$ \\
3 & 0.2479 & 8 & $7.747410^{-3}$ \\
4 & 0.1240 & 9 & $3.873710^{-3}$ \\
5 & $6.197910^{-2}$ & 10 & $1.936810^{-3}$ \\
\hline
\end{tabular}

Example 2. In the next example we consider the following system of equations

$$
\begin{aligned}
& u_{1}(x)=x^{2}-\frac{10 x^{3}}{3}+\frac{x^{4}}{6}+2\left(\int_{0}^{x}(t-x) u_{1}(t) d t+\int_{0}^{x}(t+x) u_{2}(t) d t\right), \\
& u_{2}(x)=2 x+\frac{2 x^{3}}{3}-\frac{7 x^{4}}{6}+2\left(\int_{0}^{x}(t+x) u_{1}(t) d t+\int_{0}^{x}(t-x) u_{2}(t) d t\right)
\end{aligned}
$$

for $x \in[0,1]$. Solution of the above system is given by functions

$$
u_{d 1}(x)=x^{2}, \quad u_{d 2}(x)=2 x .
$$


In this case we have $M_{K}=5$ and $\|R\|=1$. It means that this time condition (2.7) is not satisfied. Theorem 3 gives only the sufficient condition for the convergence of created series. Presented example illustrates this fact. We have here an example of the system for which the condition (2.7) is not fulfilled but still, by choosing properly the value of the convergence control parameter $h$, we can assure convergence of the method.

In this example the approximate location of the effective region of the convergence control parameter is equal to $\mathbf{R}_{h}=(-1.3,0)$ (see Figure $5 \mathrm{~b}$ ). This time the $h$-curve gives the interval $(-1.8,-0.2)$ (see Figure $5 \mathrm{a})$.

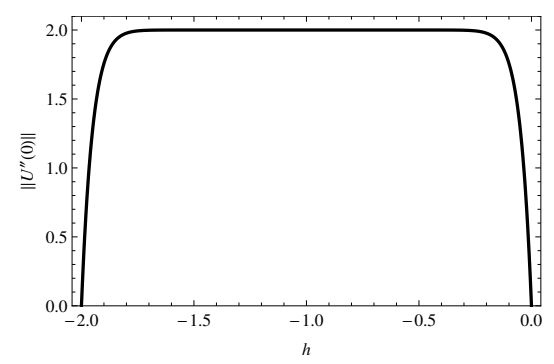

a)

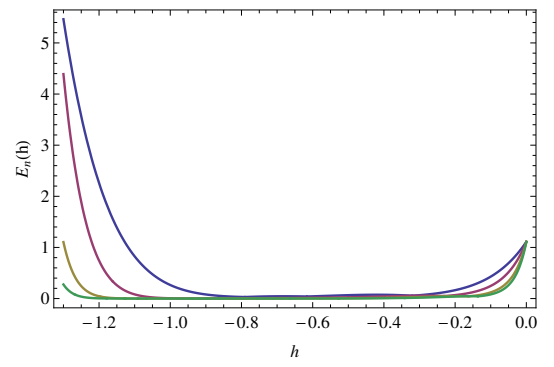

b)

Figure 5. The $h$-curve $(a)$ and squared residual $E_{n}(h)(b)$ for $n=3,5,8,10$ (the successive curves from the top)

Taking the optimum value of the convergence control parameter equal to $-1(h=-1)$ and the initial approximation in form $\mathbf{U}_{0}(x)=[0,0]^{T}$ we obtain, successively

$$
\begin{aligned}
& u_{1,1}(x)=x^{2}-\frac{10 x^{3}}{3}+\frac{x^{4}}{6}, \quad u_{2,1}(x)=2 x+\frac{2 x^{3}}{3}-\frac{7 x^{4}}{6} \\
& u_{1,2}(x)=\frac{10 x^{3}}{3}-\frac{x^{4}}{6}+\frac{14 x^{5}}{15}-\frac{13 x^{6}}{15} \\
& u_{2,2}(x)=-\frac{2 x^{3}}{3}+\frac{7 x^{4}}{6}-\frac{46 x^{5}}{15}+\frac{x^{6}}{5} \\
& u_{1,3}(x)=-\frac{14 x^{5}}{15}+\frac{13 x^{6}}{15}-\frac{68 x^{7}}{35}+\frac{29 x^{8}}{210}, \\
& u_{2,3}(x)=\frac{46 x^{5}}{15}-\frac{x^{6}}{5}+\frac{76 x^{7}}{105}-\frac{33 x^{8}}{70}, \\
& u_{1,4}(x)=\frac{68 x^{7}}{35}-\frac{29 x^{8}}{210}+\frac{374 x^{9}}{945}-\frac{191 x^{10}}{945}, \\
& u_{2,4}(x)=-\frac{76 x^{7}}{105}+\frac{33 x^{8}}{70}-\frac{886 x^{9}}{945}+\frac{13 x^{10}}{189}, \ldots
\end{aligned}
$$

Hence we receive the following approximate solution

$$
\begin{aligned}
& \widehat{u}_{1, n}(x)=x^{2}+a_{n} x^{2 n+1}+b_{n} x^{2 n+2}, \\
& \widehat{u}_{2, n}(x)=2 x+c_{n} x^{2 n+1}+d_{n} x^{2 n+2},
\end{aligned}
$$


where $\left(a_{n}\right),\left(b_{n}\right),\left(c_{n}\right)$ and $\left(d_{n}\right)$ are sequences converging to zero. In Table 3 there are presented the errors $\left(\left\|u_{d i}-\widehat{u}_{i, n}\right\|=\sup _{x \in[0,1]}\left|u_{d i}(x)-\widehat{u}_{i, n}(x)\right|\right)$ occuring in the approximations of exact solution by the successive approximate solutions for $h=-1$. Whereas, distributions of error in the entire interval $[0,1]$ for $n=20$ are displayed in Figure 6 .

Table 3. Errors of approximations of the exact solution

\begin{tabular}{llllll}
\hline$n$ & $\left\|u_{d 1}-\widehat{u}_{1, n}\right\|$ & $\left\|u_{d 2}-\widehat{u}_{2, n}\right\|$ & $n$ & $\left\|u_{d 1}-\widehat{u}_{1, n}\right\|$ & $\left\|u_{d 2}-\widehat{u}_{2, n}\right\|$ \\
\hline 1 & 3.1667 & 0.5000 & 11 & $6.007610^{-5}$ & $2.927410^{-5}$ \\
2 & $9.055310^{-2}$ & 2.8667 & 12 & $5.039010^{-6}$ & $9.941110^{-6}$ \\
3 & 1.8048 & 0.2524 & 13 & $1.519710^{-6}$ & $7.969910^{-7}$ \\
4 & 0.1937 & 0.8688 & 14 & $1.166310^{-7}$ & $2.158810^{-7}$ \\
5 & 0.3381 & $9.668110^{-2}$ & 15 & $2.863910^{-8}$ & $1.588210^{-8}$ \\
6 & $3.707410^{-2}$ & 0.1104 & 16 & $2.022810^{-9}$ & $3.563510^{-9}$ \\
7 & $3.102610^{-2}$ & $1.168510^{-2}$ & 17 & $4.175010^{-10}$ & $2.420010^{-10}$ \\
8 & $3.143710^{-3}$ & $7.652910^{-3}$ & 18 & $2.729810^{-11}$ & $4.621210^{-11}$ \\
9 & $1.681710^{-3}$ & $7.398110^{-4}$ & 19 & $4.847510^{-12}$ & $2.913110^{-12}$ \\
10 & $1.549610^{-4}$ & $3.331510^{-4}$ & 20 & $2.949610^{-13}$ & $4.831810^{-13}$ \\
\hline
\end{tabular}

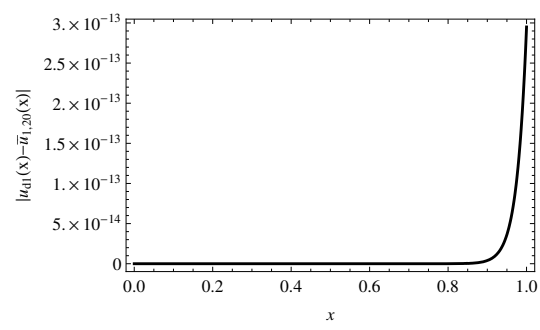

a)

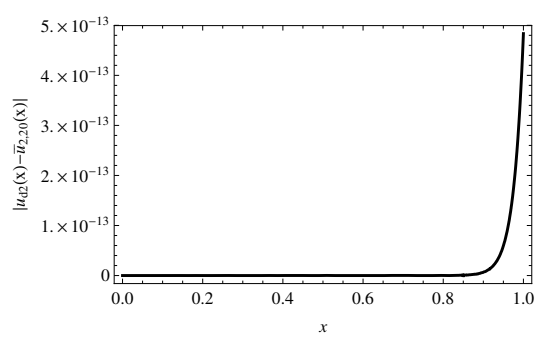

b)

Figure 6. Distribution of error of the exact solution approximation for $n=20$

In this example the errors are monotonically decreasing from $n=7$. Therefore to achieve a similar approximation, just like in the previous example, we need to do a few more iterations. By selecting the convergence control parameter $h$ from the effective region $\mathbf{R}_{h}$ we always receive a convergent series, however for $h \neq-1$ the rate of convergence is smaller. For $h=-1.25$ the approximation error $\widehat{u}_{i, 20}$ is equal to $1.86268 \cdot 10^{-3}$, for $h=-1.1$ the same approximation error is $1.9188 \cdot 10^{-7}$ and for $h=-0.75$ it is equal to $7.6025 \cdot 10^{-6}$, however for $h=-0.2$ it is equal to 0.3380 . For the value outside of the effective region the errors increase together with the increasing number of iterations. According to Remark 2 , for $h=-1$ in case of the Volterra equation we have certainly that the series will be convergent. These calculations show that for other value of the convergence control parameter from the effective region $\mathbf{R}_{h}$ the series is convergent even though the condition (2.7) is not satisfied. 
Example 3. In the last example we discuss the following system of equations

$$
\begin{aligned}
& u_{1}(x)=x-6+16 e^{-1}+\int_{0}^{1} e^{-t} u_{1}(t) u_{2}(t) d t \\
& u_{2}(x)=x^{2}-24+65 e^{-1}+\int_{0}^{1} e^{-t}\left(u_{1}(t)\right)^{2} u_{2}(t) d t
\end{aligned}
$$

for $x \in[0,1]$, solution of which is given by functions

$$
u_{d 1}(x)=x, \quad u_{d 2}(x)=x^{2} .
$$

In this case the condition (3.4) is not satisfied, therefore we cannot conclude on this ground that the system possesses exactly one solution (see Theorem 5).

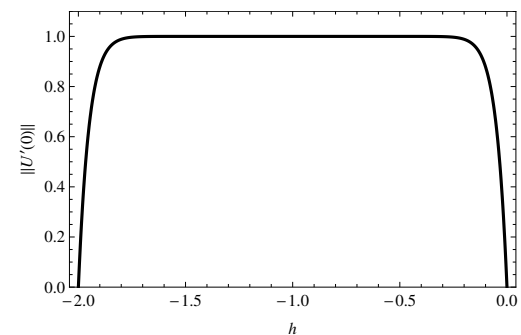

a)

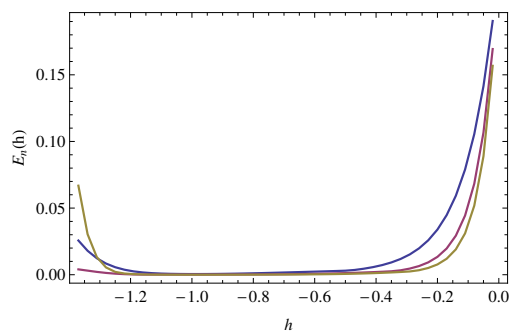

b)

Figure 7. The $h$-curve $(a)$ and squared residual $E_{n}(h)(b)$ for $n=5,8,10$ (the successive curves from the top)

Now the optimum value of the convergence control parameter $h$ is equal to -0.47 . The $h$-curve and square residual are presented in Figure 7 . As the initial approximation we take $\mathbf{U}_{0}(x)=[0,0]^{T}$. For $n=2$ we receive

$$
\widehat{u}_{1,2}(x)=-0.0819263+0.7191 x, \quad \widehat{u}_{2,2}(x)=-0.0631631+0.7191 x^{2},
$$

for $n=10$ we obtain

$$
\widehat{u}_{1,10}(x)=-0.033142+0.998251 x, \quad \widehat{u}_{2,10}(x)=-0.0408886+0.998251 x^{2},
$$

whereas for $n=20$ we get

$$
\widehat{u}_{1,20}(x)=-0.0078125+0.999997 x, \quad \widehat{u}_{2,20}(x)=-0.015625+0.999997 x^{2} .
$$

Table 4 presents the errors $\left(\left\|u_{d i}-\widehat{u}_{i, n}\right\|=\sup _{x \in[0,1]}\left|u_{d i}(x)-\widehat{u}_{i, n}(x)\right|\right)$ which occur in approximating the exact solution by the successive approximate solutions.

Table 5 presents several values of the norm of functions $\mathbf{U}_{m}$ for the optimal value of the convergence control parameter. It can be noticed that they form a strictly decreasing sequences. That, according to Theorem 7 , can be treated as the indicator of convergence of the series in (1.4). 
Table 4. Errors of approximations of the exact solution

\begin{tabular}{cccccc}
\hline$n$ & $\left\|u_{d 1}-\widehat{u}_{1, n}\right\|$ & $\left\|u_{d 2}-\widehat{u}_{2, n}\right\|$ & $n$ & $\left\|u_{d 1}-\widehat{u}_{1, n}\right\|$ & $\left\|u_{d 2}-\widehat{u}_{2, n}\right\|$ \\
\hline 1 & 0.583547 & 0.571283 & 11 & 0.029441 & 0.036087 \\
2 & 0.362826 & 0.344063 & 12 & 0.025160 & 0.030755 \\
3 & 0.237669 & 0.223637 & 13 & 0.021704 & 0.026376 \\
4 & 0.162669 & 0.157155 & 14 & 0.018853 & 0.022752 \\
5 & 0.115839 & 0.117697 & 15 & 0.016461 & 0.019730 \\
6 & 0.085636 & 0.092175 & 16 & 0.014431 & 0.017195 \\
7 & 0.065566 & 0.074310 & 17 & 0.012696 & 0.015059 \\
8 & 0.051809 & 0.061036 & 18 & 0.011220 & 0.013192 \\
9 & 0.042055 & 0.050772 & 19 & 0.009903 & 0.011376 \\
10 & 0.034891 & 0.042637 & 20 & 0.008925 & 0.010033 \\
\hline
\end{tabular}

Table 5. Values of the norm of functions $\mathbf{U}_{m}$ for the optimal value of the convergence control parameter

\begin{tabular}{clclllll}
\hline$m$ & $\left\|\mathbf{U}_{m}\right\|$ & $m$ & $\left\|\mathbf{U}_{m}\right\|$ & $m$ & $\left\|\mathbf{U}_{m}\right\|$ & $m$ & $\left\|\mathbf{U}_{m}\right\|$ \\
\hline 1 & 0.428717 & 6 & 0.030203 & 11 & 0.006550 & 16 & 0.002537 \\
2 & 0.227220 & 7 & 0.020070 & 12 & 0.005332 & 17 & 0.002139 \\
3 & 0.125158 & 8 & 0.013757 & 13 & 0.004379 & 18 & 0.001817 \\
4 & 0.075000 & 9 & 0.010263 & 14 & 0.003624 & 19 & 0.001307 \\
5 & 0.046829 & 10 & 0.008135 & 15 & 0.003022 & 20 & 0.001247 \\
\hline
\end{tabular}

\section{Conclusions}

Discussion on the systems of integral equations, the linear as well as the nonlinear ones, presented in Sections 2 and 3 can be transferred "almost" without changes onto the more general case in which the interval $[a, b]$ is replaced, for example, by a compact metric and simultaneously measurable space $\Omega$ (or in more general cases by a metric space and by a measurable space). The integral is taken then over the sets with common bounded measure and so on. In this case some convergence aspects, like for instance the uniqueness of solution, would need the application of, for example, the Schauder theorem and the Schauder modified theorem $[8,22]$. Considerations of that kind will be the subject of our future papers.

\section{Acknowledgement}

The Authors would like to thank Professor Roman Wituła for valuable discussion enabling to give the final shape to this paper. The Authors appreciate also the help given by Edyta Sporysz in the editing stage of this paper preparation.

The Authors would like to express their thanks to all the Referees for the dedicated time and valuable remarks enabling to improve the paper. 


\section{References}

[1] S. Abbasbandy and E. Shivanian. A new analytical technique to solve Fredholm's integral equations. Numerical Algorithms, 56(1):27-43, 2010. http://dx.doi.org/10.1007/s11075-010-9372-2.

[2] G.A. Afroozi, J. Vahidi and M. Saeidy. Solving a class of two-dimensional linear and nonlinear Volterra integral equations by means of the homotopy analysis method. International Journal of Nonlinear Science, 9(2):213-219, 2010.

[3] F.T. Akyildiz and K. Vajravelu. Magnetohydrodynamic flow of a viscoelastic fluid. Physics Letters A, 372(19):3380-3384, 2008. http://dx.doi.org/10.1016/j.physleta.2008.01.073.

[4] F. Awawdeh, A. Adawi, and S. Al-Shara'. A numerical method for solving nonlinear integral equations. International Mathematical Forum, 4(17):805-817, 2009 .

[5] F. Bazrafshan, A.H. Mahbobi, A. Neyrameh, A. Sousaraie and M. Ebrahimi. Solving two-dimensional integral equations. Journal of King Saud University Science, 23(1):111-114, 2011. http://dx.doi.org/10.1016/j.jksus.2010.06.017.

[6] Sh.S. Behzadi. Solving fuzzy nonlinear Volterra-Fredholm integral equations by using homotopy analysis and Adomian decomposition methods. Journal of Fuzzy Set Valued Analysis, 2011:1-13, 2011. http://dx.doi.org/10.5899/2011/jfsva00067.

[7] Y. Cherruault. Convergence of Adomian's method. Kybernetes, 18(2):31-38, 1989. http://dx.doi.org/10.1108/eb005812.

[8] K. Goebel and W.A. Kirk. Topics in Metric Fixed Point Theory. Cambridge University Press, Cambridge, 1990. http://dx.doi.org/10.1017/CBO9780511526152.

[9] E. Hetmaniok, I. Nowak, D. Słota and R. Wituła. A study of the convergence of and error estimation for the homotopy perturbation method for the VolterraFredholm integral equations. Applied Mathematics Letters, 26(1):165-169, 2013. http://dx.doi.org/10.1016/j.aml.2012.08.005.

[10] E. Hetmaniok, I. Nowak, D. Słota and R. Wituła. Convergence and error estimation of homotopy analysis method for some type of nonlinear and linear integral equations. Journal of Numerical Mathematics, 23(4):331-344, 2015. http://dx.doi.org/10.1515/jnma-2015-0022.

[11] E. Hetmaniok, D. Słota, T. Trawiński, and R. Wituła. Usage of the homotopy analysis method for solving the nonlinear and linear integral equations of the second kind. Numerical Algorithms, 67(1):163-185, 2013. http://dx.doi.org/10.1007/s11075-013-9781-0.

[12] E. Hetmaniok, D. Słota and R. Wituła. Convergence and error estimation of homotopy perturbation method for Fredholm and Volterra integral equations. Applied Mathematics and Computation, 218(21):10717-10725, 2012. http://dx.doi.org/10.1016/j.amc.2012.04.041.

[13] S. Liao. Homotopy analysis method: a new analytic method for nonlinear problems. Applied Mathematics and Mechanics, 19(10):957-962, 1998. http://dx.doi.org/10.1007/BF02457955.

[14] S. Liao. Beyond Perturbation: Introduction to the Homotopy Analysis Method. Modern Mechanics and Mathematics. Chapman and Hall/CRC Press, Boca Raton, 2003. 
[15] S. Liao. On the homotopy analysis method for nonlinear problems. Applied Mathematics and Computation, 147(2):499-513, 2004. http://dx.doi.org/10.1016/S0096-3003(02)00790-7.

[16] S. Liao. Notes on the homotopy analysis method: some definitions and theorems. Communications in Nonlinear Science and Numerical Simulation, 14(4):983997, 2009. http://dx.doi.org/10.1016/j.cnsns.2008.04.013.

[17] S. Liao. Homotopy Analysis Method in Nonlinear Differential Equations. $\quad$ Springer/Higher Education Press, Berlin/Beijing, 2012. http://dx.doi.org/10.1007/978-3-642-25132-0.

[18] A. Molabahrami, A. Shidfar and A. Ghyasi. An analytical method for solving linear Fredholm fuzzy integral equations of the second kind. Computers \& Mathematics with Applications, 61(9):2754-2761, 2011. http://dx.doi.org/10.1016/j.camwa.2011.03.034.

[19] Z.M. Odibat. A study on the convergence of homotopy analysis method. Applied Mathematics and Computation, 217(2):782-789, 2010. http://dx.doi.org/10.1016/j.amc.2010.06.017.

[20] A. Shayganmanesh. Generalizing homotopy analysis method to solve system of integral equations. Journal of Mathematical Extension, 5(1):21-30, 2010.

[21] A. Shidfar and A. Molabahrami. Solving a system of integral equations by an analytic method. Mathematical and Computer Modelling, 54(1-2):828-835, 2011. http://dx.doi.org/10.1016/j.mcm.2011.03.031.

[22] D.R. Smart. Fixed Point Theorems. Cambridge University Press, London-New York, 1974.

[23] M. Turkyilmazoglu. Convergence of the homotopy analysis method. arXiv:1006.4460v1, pp. 1-12, 2010.

[24] S. Vahdati, Z. Abbas and M. Ghasemi. Application of homotopy analysis method to Fredholm and Volterra integral equations. Mathematical Sciences, 4(3):267282,2010 .

[25] H. Vosughi, E. Shivanian and S. Abbasbandy. A new analytical technique to solve Volterra's integral equations. Mathematical Methods in the Applied Sciences, 34(10):1243-1253, 2011. http://dx.doi.org/10.1002/mma.1436.

[26] K. Yabushita, M. Yamashita and K. Tsuboi. An analytic solution of projectile motion with the quadratic resistance law using the homotopy analysis method. Journal of Physics A: Mathematical and Theoretical, 40(29):8403-8416, 2007. http://dx.doi.org/10.1088/1751-8113/40/29/015. 